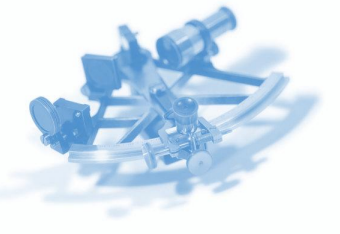

\author{
Stuart Freake \\ Department of Physics and \\ Astronomy \\ The Open University \\ Walton Hall \\ Milton Keynes \\ MK7 6AA \\ s.m.freake@open.ac.uk
}

The majority of Open

University courses include

summative tutor marked

assignments (TMAs),

which are submitted by

students at intervals

during the presentation of a course.

\section{Electronic marking of physics assignments using a Tablet PC}

\begin{abstract}
Producing and marking assignments electronically has advantages for both students and academic staff. However, marking and commenting on electronic assignments requires a simple and efficient method for annotating students' scripts. We report here on a pilot project that provided associate lecturers (ALs) for a Level 2 Open University physics course with Tablet PCs and appropriate software that enabled them to input handwritten annotation, including equations and diagrams, to files containing students' solutions to assignments. Surveys of the views of ALs indicated that this method of electronic marking is effective and acceptable to students and to a large majority of ALs. It could be used to streamline assignment handling and marking procedures in campus universities as well as in distance learning contexts.
\end{abstract}

\section{Introduction}

The majority of Open University courses include summative tutor marked assignments (TMAs), which are submitted by students at intervals during the presentation of a course. The TMAs are marked by associate lecturers (ALs, also known as tutors), who are based in different locations around the country and are each responsible for a group of about 25 students. The traditional method of producing and marking TMAs is paper based: a student writes their answers to the assignment questions on paper (or word-processes them and prints them out), they use the postal system to mail the assignment to their $A L$, who marks and comments on the assignment by annotating the student's script. The AL sends marked assignments to the Open University in Milton Keynes, where the marks are recorded in a database, a sample of scripts are copied so that each AL's marking can be monitored, and the scripts are then posted back to students. This system involves shuffling large quantities of paper around the country and there are delays, expense and inconvenience associated with the postal system, with the transcription of marks and with the copying of assignments for monitoring. Now that OU students and ALs are expected to have access to a computer and the internet, some of the delays, costs and inconvenience can be reduced by handling TMAs electronically.

The OU has developed a system for handling electronic tutor marked assignments (eTMAs), and is encouraging course teams and students to use this system wherever possible. The eTMA system allows students to submit electronic files containing their assignments over the internet to a file handling system on a server in Milton Keynes. ALs download the eTMA files for their students from the server, mark and comment on the eTMAs offline, and then return the marked scripts over the internet to the server in Milton Keynes, where marks are automatically recorded in the appropriate database and a sample of scripts is made available for monitoring. Students can then download their marked assignment from the central server. This system has many advantages for students, ALs and the OU over the paper-based assignment system that relies on the post office for moving the assignments around the country.

One advantage is that the eTMA system in principle allows ALs to give more rapid feedback to students following the cut-off date for the assignment than does the paperbased system. Gibbs and Simpson ${ }^{1}$ established a list of conditions under which assessment effectively supports learning. A key finding of this work was that it is essential that "feedback is timely in that it is received by students while it still matters to them and in time for them to pay attention to further learning or receive further assistance".

However, the benefits of rapid feedback could be more than offset if a longer time were required for ALs to mark eTMAs to the same standard as paper TMAs, so that the quality and quantity of feedback provided to students were reduced. Assignments for physics and astronomy courses generally involve large amounts of mathematical notation, 
equations and diagrams, and when marking eTMAs, ALs need to be able to insert equations and diagrams, and the time required to do this is generally the major issue. Keyboard and mouse based tools, such as equation editors and drawing programs, are much more time consuming than annotating a paper script with a pen. The pilot project described in this report was set up to investigate ways in which ALs could mark physics eTMAs easily, and could incorporate equations and diagrams just as they would if they were marking a paper assignment.

\section{The project}

In March 2006 the Physics Innovations Centre for Excellence in Teaching and Learning ( $\pi \mathrm{CETL})$ at the Open University agreed to fund a pilot project to investigate the use of Tablet PCs by ALs for marking assignments. All of the ALs who were tutoring a Level 2 physics course, S207 The physical world, were invited to participate in the project, and 19 of the 24 ALs agreed to take part. The participating ALs were provided with a Tablet PC (Toshiba Tecra M4), with appropriate software installed to enable them to add handwritten annotation to a student's eTMA file. The following year (2007) all ALs for the course participated in the electronic marking project. Students were informed about the benefits of using the eTMA system, but they had the options of submitting assignments electronically or on paper.

\section{Marking procedures with the Tablet PC}

ALs had a number of options for marking and commenting on eTMAs using the Tablet PC.

\section{Ink annotation of a pdf file using PDF Annotator}

We wanted to allow students to produce assignments using whatever application they found convenient, but wanted ALs to be able to use a single application for marking. We therefore recommended that ALs used pdf format for marking. The Tablet PCs were sent out with PDF Creator ${ }^{2}$ installed, and this application creates a 'virtual printer' that can convert into pdf format any student file that can be opened and printed from the AL's Tablet PC. The AL simply opens a student's assignment file - which requires that an appropriate application is installed on the Tablet PC - selects Print and chooses PDF Creator as the printing device. 'Printing' the file then produces a pdf version of the file, rather than printing out on paper. PDF Creator allows the user to combine a number of different files into a single pdf file.

The AL then opens the pdf file from the PDF Annotator ${ }^{3}$ application. With the computer in the tablet configuration, the pdf file can be annotated by writing on the screen with the tablet pen in exactly the same way that a paper assignment would be annotated with a conventional pen, but with the advantages that it is straightforward to:
- change pen colour and line width - so it's possible to colour code different types of comments;

- erase or correct comments;

- move or resize comments;

- save comments as macros that can then be inserted easily elsewhere in the same document or in other documents;

- insert blank pages for additional comments, or to append files (such as specimen answers).

When the AL has finished marking, they 'melt' the annotation so that it becomes a permanent part of the document, they save the file in pdf format and follow the standard procedures for returning the eTMA to the OU server. The student
Figure 1: An example of a tutor's annotation on a page from an assignment The tutor's comments would be much clearer to a student viewing a coloured image on their monitor.

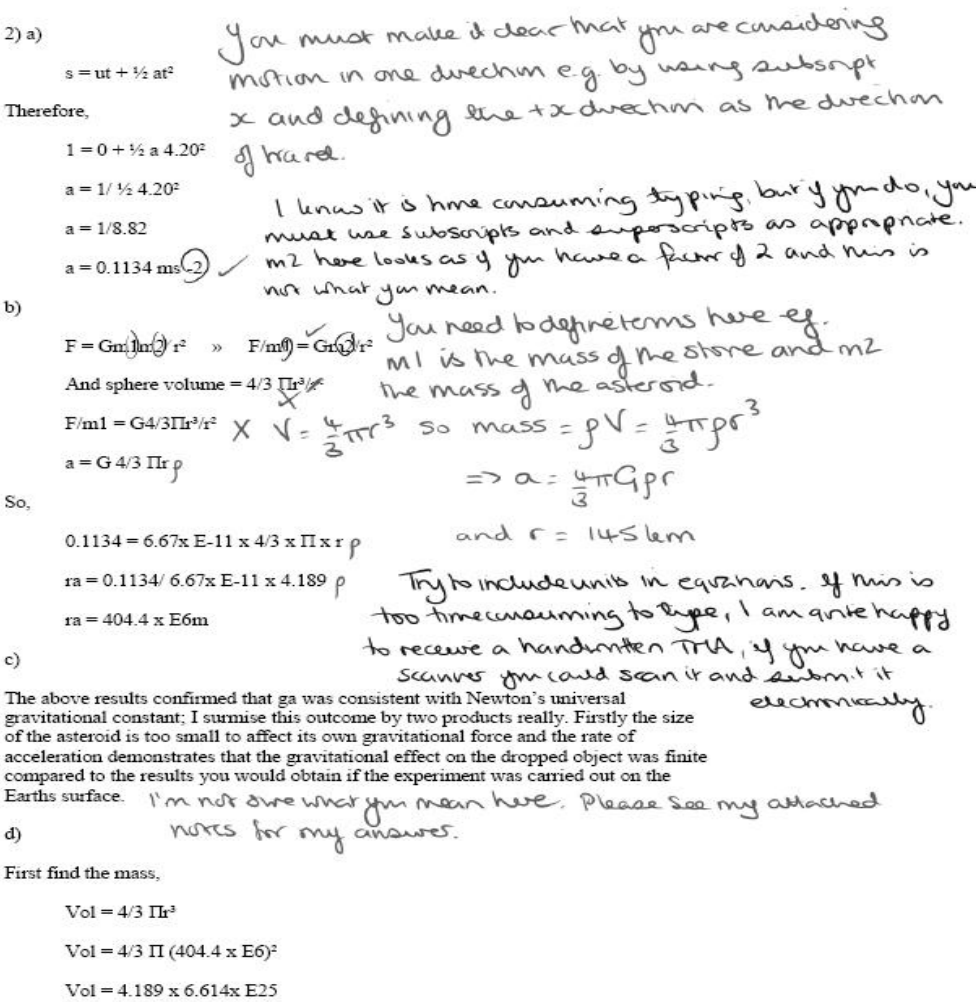

downloads the marked assignment from the server and opens the file using Adobe Reader. An example of a page from a marked assignment is shown in Figure 1.

PDF Annotator also allows simple text to be keyed into a text box. However, mixing keyboard entry and tablet pen entry is not particularly easy, since it involves switching back and forth between using the laptop configuration and using the tablet configuration. Switching between the two configurations of the screen takes about 15 seconds, which is not an unreasonable overhead, but the switchover is somewhat clumsy. An alternative procedure used by some ALs is to connect a USB keyboard to the Tablet PC, so that both keyboard entry and tablet pen entry are possible in the tablet configuration. 


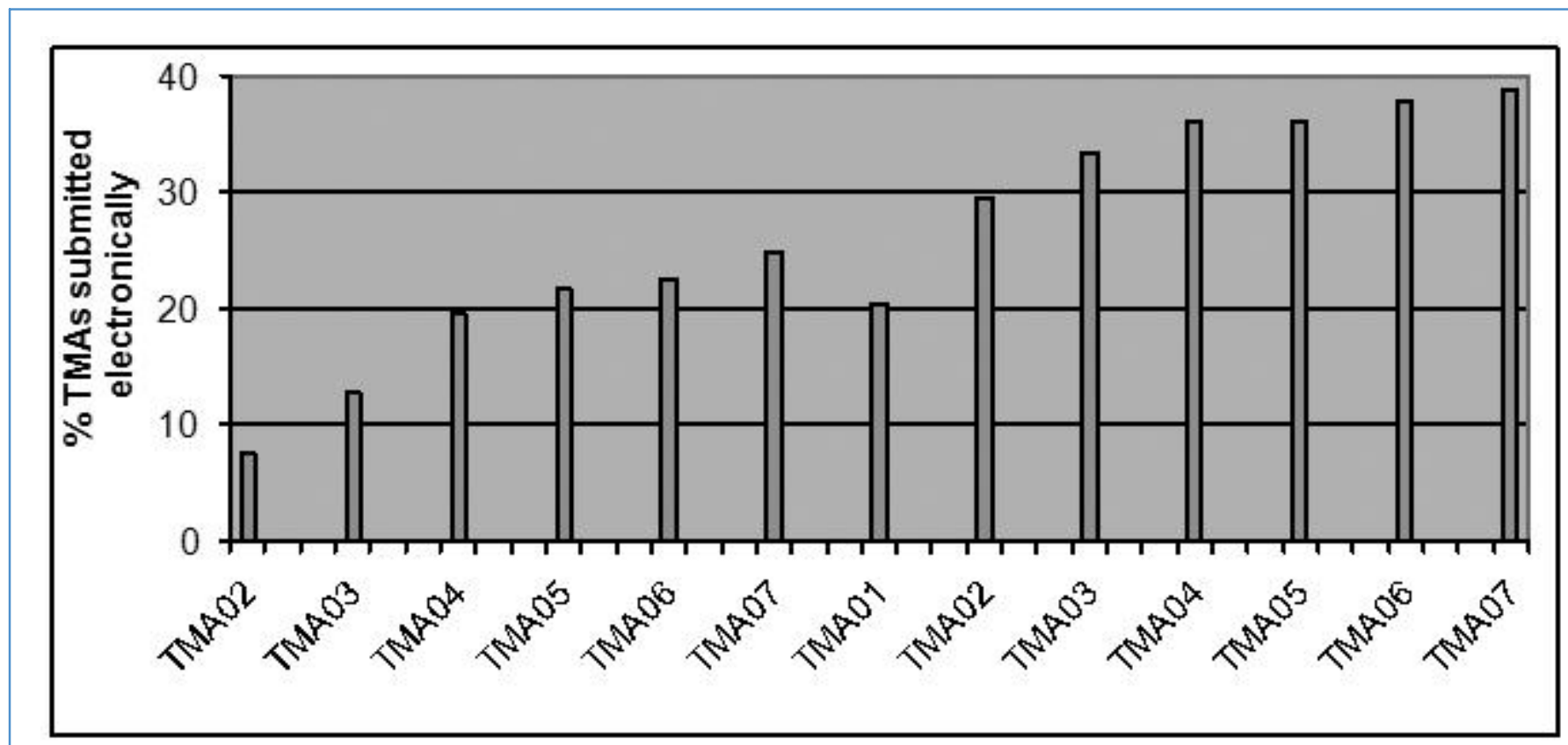

Figure 2: Percentage of TMAs submitted electronically for each assignment during 2006 (shown on the left) and 2007 (shown on the right).

Ink annotation using Microsoft Word

A large majority of the eTMAs were submitted by students as Word documents, and so a second option for eTMA marking is for ALs to use the Ink tools available in the Tablet PC version of Word 2003, which was installed on these computers. The Ink Annotation tool can be used to write with the tablet pen anywhere in an open Word document, and, as with PDF Annotator, it is possible to change pen colour and line width, to erase ink marks, and to reposition and resize comments. It is also possible to use the Ink Comment tool to insert comments in the margin. This is similar to using the normal Insert Comment feature in Word, except that the comments are handwritten in a balloon in the margin using the tablet pen, and these comments can include equations and diagrams. As with PDF Annotator, it is possible to switch back and forth between using the keyboard to enter simple text in the laptop configuration and using the tablet pen to enter equations and figures in the tablet configuration. When the AL has finished marking the script, the file is saved in .doc format for return to the student via the eTMA system.

Ink annotation using Windows Journal

A third way to mark eTMAs with a Tablet PC is to convert the student's file to a Windows Journal file and then to annotate this file using the tablet pen. As with PDF Creator, Windows Journal installs a virtual printer with which any printable file submitted by a student can be converted to a Windows Journal file (.jnt), and this file can then be annotated in much the same way as a pdf file is annotated using PDF Annotator. This option was not suggested to the physics ALs, and none of them apparently discovered or used this method, but this was the approach used by Fisher ${ }^{4}$ in another Open University Tablet PC trial with a technology course.

\section{Evaluation}

After completion of the 2006 presentation of S207, a brief questionnaire was emailed to the ALs who had participated in the pilot project, and 15 of the 19 participants responded. A more comprehensive questionnaire was emailed at the end of 2007 to the 24 ALs involved in that year's presentation, and 23 of them responded. For both years, information about the number of paper TMAs and eTMAs marked by each AL for each of the course assignments was obtained from the assignment handling database.

\section{Percentage of eTMAs submitted electronically}

The percentage of the total number of TMAs that were submitted electronically grew steadily during the project, as shown in Figure 2, and reached $40 \%$ by the end of 2007 .

\section{Format of the eTMA files submitted}

ALs reported that $(70 \%)$ of the eTMAs submitted in 2007 were word-processed, including the equations and diagrams. About $20 \%$ were largely word-processed, but with equations and/or diagrams produced by hand, and scanned images inserted into the document. The other $10 \%$ of eTMAs were completely handwritten and then scanned. Most of the eTMAs (83\%) were submitted as Word .doc files, but small percentages were submitted in other word-processor formats, as pdf files or as image files.

\section{Marking procedures used by ALs}

In 2007 the ALs were approximately equally split between those who converted files to pdf format and then marked using PDF Annotator, and those who marked using Word. Most of the ALs who opted to use Word for marking did not receive any files in other formats, and this may have been because they specifically asked students to submit eTMAs as .doc files. A variety of approaches were used when marking in Word; most ALs used both keyboard and tablet pen entry, but a couple opted not to use the Tablet PC and used a conventional computer for inserting text boxes and comments (using Track Changes). None of the ALs used the Windows Journal application. 


\section{Difficulties in learning how to use the marking system} ALs were asked whether they had problems learning how to use the Tablet PC or the associated software. Even though the only information that they had received was the standard user manuals for the computer and the software, there were few reported problems. A few tutors said they would have appreciated more guidance on the conversion of files to pdf format and on the use of PDF Annotator, and one commented that the use of the Print File command in PDF Creator to produce a pdf file was counterintuitive. A couple of ALs thought there should be explicit guidance about how to insert pages into a script for additional comments. During 2007 we produced a document that provided guidance on the use of the Tablet PC for marking and this was distributed to all participating ALs. This document will be available in future years for ALs who are new to this method of marking.

\section{Ergonomic considerations}

Six tutors said that it took them some time to find a comfortable way to mark using the Tablet PC. Three of them found that it was difficult to use the Tablet PC while wearing varifocal glasses, and two of them overcame this problem by using single prescription lenses while marking. Two ALs worked with the Tablet PC on their laps and another found that they had to use a lower working surface. One AL found using the Tablet PC so uncomfortable that he minimised use of it in the tablet mode by connecting an external monitor, keyboard and mouse to the Tablet PC and only using pen input for equations or diagrams that could not be copied or referred to. In future it would be appropriate to provide some 'ergonomic' advice as part of the guidance to ALs on the use of the Tablet PC for eTMA marking.

\section{Advantages of using the Tablet PC}

The ALs were asked about the advantages of using the Tablet $\mathrm{PC}$ for electronic marking compared with paper marking, and the main points they mentioned were:

- the ability to annotate eTMAs in the same way that they would annotate paper TMAs;

- the ability to erase comments, or to revise them; they were pleased that they did not need to use correction fluid or to cross out comments; they thought their comments were often more considered, since they could be changed if necessary after reading a later part of the answer;

- no longer needing to contend with the piles of paper associated with conventional TMA marking; also, the pages can't get out of order;

$\checkmark \quad$ the ability to copy and paste comments between different TMAs;

- comments could be more legible - though it may be necessary to write more slowly, or to rewrite comments;

- the ease of inserting relevant parts of the specimen answers:

- several ALs said that eTMA marking requires less time than conventional marking;

automated addition of marks avoids errors;

- improved turnaround time, particularly for students outside the UK;

- avoids use of the paper-based mail delivery system; it is easier to upload marked eTMAs than to post paper TMAs; eTMAs can be accessed anywhere that there is a broadband connection;

- $\mathrm{AL}$ retains copy of assignment on their computer for reference.

\section{Ongoing problems and disadvantages of using the Tablet $P C$ for marking}

In addition to the ergonomic considerations noted above, the main ongoing problems and disadvantages mentioned were:

- some ALs found that it took longer to mark assignments electronically;

- converting files to pdf format was tedious;

- it was difficult to trawl through pages to get an overview to ensure that the comments were appropriate, or difficult to lay out pages and compare several scripts to ensure consistency;

- it was inconvenient to switch between different students' files when marking each question as a batch;

- it was not as convenient as paper for marking while waiting in the car

- ALs found that switching between laptop and tablet modes was inconvenient; some ALs avoided this inconvenience by using an external keyboard connected to a USB port on the Tablet PC;

- it is not possible to cut and paste in PDF Annotator;

- the reliability of electronic submission (compared with postal submission) meant that many students delayed eTMA submission until just before the deadline.

\section{Student feedback}

The small amount of feedback that ALs received from students who submitted eTMAs was positive, with most of the comments about the quick turnaround and the ability to submit right up to the cut off time. However, some students did also comment on the clarity and legibility of the comments that ALs made in the returned file.

\section{Overall views about marking eTMAs compared with marking paper TMAs}

In 2007, eleven of the 23 ALs expressed a preference for marking eTMAs and eight ALs either said explicitly that they were equally happy with paper TMAs and eTMAs or expressed no preference. Only four ALs indicated a preference for paper marking, and three of these had marked very few eTMAs. Almost all of the ALs were very positive about the pilot project. There were some reservations from those who found that eTMAs took longer to mark - this needs to be reviewed when ALs are more familiar with using this method of marking. A more detailed report on the electronic marking trial can be found on the $\pi$ CETL website ${ }^{5}$.

\section{Future work}

In 2006 , about $17 \%$ of assignments submitted to ALs taking part in the project were eTMAs, and this increased to $32 \%$ in 2007. The main reason that the majority of S207 students prefer paper submission is that they lack the IT skills to incorporate equations and diagrams into a word-processed TMA and/or find that producing an eTMA is unacceptably time -consuming. During the 2007 presentation, another $\pi$ CETL project attempted to increase the number of students submitting electronically by offering students the loan of either a scanner or a digital pen. These systems allow the student to write their assignment answers on paper, but then to convert it to a digital format that can be submitted via the eTMA system.

In 2008 we have extended the electronic marking project to two Level 3 physics and astronomy courses. 


\section{Electronic marking with campus-based students}

The OU project investigated the use of electronic marking in a distance-learning context, where a major benefit of electronic marking is that electronic transmission of assignments eliminates dependence on the postal service. This particular benefit is not relevant for campus-based students, where paper assignments can be placed in appropriate pigeonholes, but there would still be benefits from using an electronic system that recorded submission and return dates for assignments, that stored marks, and that kept copies of all marked assignments. Academics would also benefit from the elimination of piles of paper assignments, from the ability to download, mark and return assignments anywhere that they have an internet connection, and from all of the other advantages of marking electronically discussed earlier. It would be straightforward to provide campus-based students with access to scanners that could convert handwritten assignments into a single pdf file, so that lack of skills with using word-processing software, equation editors and drawing packages would not disadvantage them.

A $\pi$ CETL project at Leicester University ${ }^{6}$ is going much further, and investigating a paperless degree. So not only are assignments being handled electronically, but also all course materials are provided electronically.

\section{Conclusion}

This S207 pilot project has shown that a Tablet PC provides an effective and acceptable method of marking physics assignments electronically - from the viewpoints of both the ALs and the students. ALs used two main methods of electronic marking - annotating pdf files using PDF Annotator and using annotating doc files using Word - and the choice was a matter of personal preference. However, it must be recognized that although Tablet PCs provide a good way of marking physics assignments, their capital cost may inhibit their widespread use for marking Open University assignments.

\section{Acknowledgements}

We gratefully acknowledge the contributions of Jonathan Underwood, who set up and ran this pilot project at the Open University until he moved to University College London, and of the associate lecturers who participated in the project and whose feedback was used as the basis of this report. This project would not have been possible without the funding and support provided by $\pi \mathrm{CETL}$, which in turn is funded by the Higher Education Funding Council for England.

\section{References}

1. Gibbs, G. and Simpson, C. (2004) Conditions under which assessment supports student learning, Learning and Teaching in Higher Education, 1, 3-31.

2. Chinery, P. and Heindörfer, F., PDF Creator software, free download from <www.pdfforge.org>.

3. Grahl Software Design, PDF Annotator software, <www.GRAHL-software.com>.

4. See COLMSCT website: Fisher, W., A critical review of the use of enabling technologies <www.open.ac.uk/colmsct/projects/wendyfisher>.

5. See $\pi$ CETL website: Freake, S., Use of Tablet PCs to mark physics assignments

<www.open.ac.uk/picetl/projects/etmamarking>.

6. See $\pi$ CETL website: Symons, S., Paperless degree project

<www.open.ac.uk/picetl/projects/paperlessdegree>

\section{The small amount of feed- back that ALs received from students who submitted eTMAs was positive, with most of the comments about the quick turnaround and the ability to submit right up to the cut off time.}

